\title{
ARTICLE
}

\section{Fingermark Analysis by Fourier Transform Infrared Microscopy Using Chemometric Tools}

\author{
Marina González*iD, Kristiane de Cássia Mariotti²,3 iD, Adriano de Araújo Gomes ${ }^{4}$ iD, Marco \\ Flôres Ferrão ${ }^{4,5}$ iD, Renata Pereira Limberger ${ }^{1,3}$ iD
}

${ }^{1}$ Departamento de Farmácia, Universidade Federal do Rio Grande do Sul, Av. Ipiranga, 2752, Azenha, 90610-000, Porto Alegre, RS, Brazil

${ }^{2}$ Grupo de Biometria, Polícia Federal, Av. Ipiranga, 1365, Azenha, 90610-093, Porto Alegre, RS, Brazil ${ }^{3}$ Instituto Nacional de Ciência e Tecnologia Forense (INCT Forense), Porto Alegre, RS, Brazil

${ }^{4}$ Departamento de Química, Universidade Federal do Rio Grande do Sul, Av. Bento Gonçalves, 9500, Agronomia, 90650-001, Porto Alegre, RS, Brazil

5Instituto Nacional de Ciência e Tecnologia em Bioanalítica (INCT Bioanalítica), Campinas, SP, Brazil

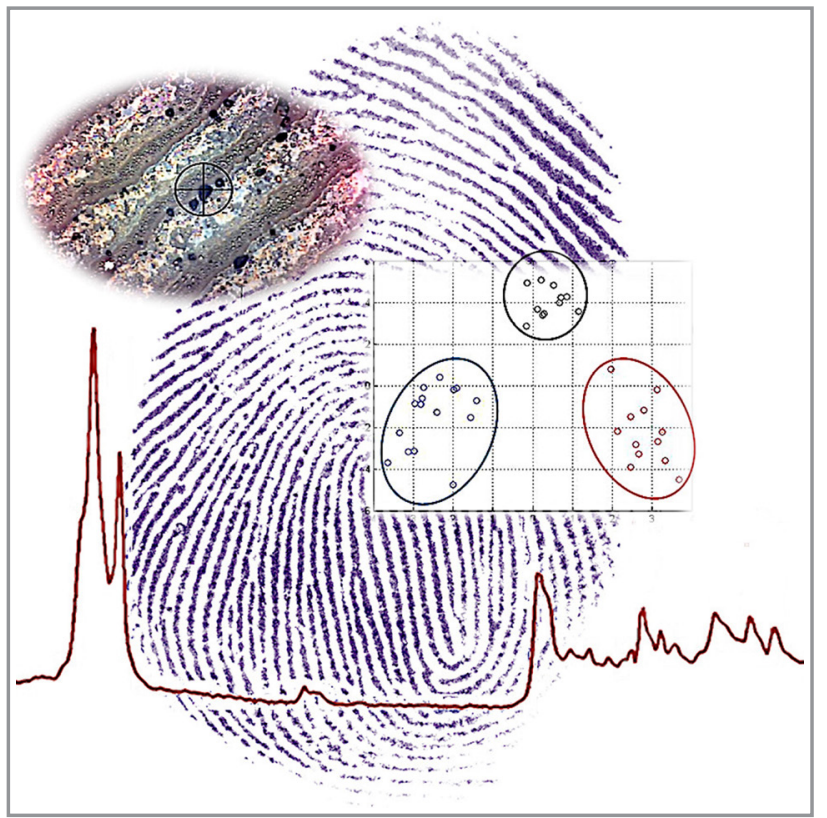

The temporal estimation of the fingermarks deposition at crime scenes is a recurring issue in forensic identification. To study this challenging topic, a preliminary study was proposed to develop a method of fingermark analysis by Fourier Transform Infrared Microscopy ( $\mu$-FTIR), using chemometric tools for time separation in a six-day aging study. The samples were collected and analyzed at hour zero, 3 days (72 hours), 4 days (96 hours), 5 days (120 hours) and 6 days (144 hours). The samples were separated into a calibration set and a test set, using Kennard Stone. Following, a comparison between variable selection tools was made of the Ant of Colony (AOC) and the Genetic Algorithm (GA) were used with subsequent application of the Linear Discriminant Analysis (LDA). The results showed that in the analyzed samples there was a predominance of sebaceous material because of the presence of saturated esters signals, with two regions of interest in the infrared spectra, the first being 1800 - $1100 \mathrm{~cm}^{-1}$ and the second region being $3000-2800 \mathrm{~cm}^{-1}$. The statistical tools could group the fingermarks by donors and by age, emphasizing the separation within the tested period. More studies need to be carried out, but this work provide that $\mu-F T I R$ associated with chemometric analysis was able to separate fingermarks samples aged for up to a week.

Keywords: forensic chemistry, latent fingerprints, aging, FTIR, chemometrics.

Cite: González, M.; Mariotti, K. C.; Gomes, A. A.; Ferrão, M. F.; Limberger, R. P. Fingermark Analysis by Fourier Transform Infrared Microscopy Using Chemometric Tools. Braz. J. Anal. Chem., 2021, 8 (32), pp 141-154. doi: http://dx.doi.org/10.30744/ brjac.2179-3425.AR-25-2021

Submitted 01 March 2021, Resubmitted 28 May 2021, Accepted 31 May 2021, Available online 08 June 2021. 


\section{INTRODUCTION}

Fingermarks are the most common vestiges in crime scenes, bringing information about an individual present who may or may not be responsible for the commission of a crime [1]. For criminal investigation, the fingermark identification remains the most widely used [2]. One of the great challenges in the fingermark analysis is the aging establishment, that is, the period elapsed between the trace deposition by the donor and the analysis or development by forensic expertise. Questions about this subject have been the object of investigation by the academy and expert forces [1-7]. Placing events and evidence in time is an essential issue in investigations and, among the typical issues of forensic science, time is generally not explored. The reason for this can be attributed to the issue complexity [4]. The latent fingermarks aging is one of the most challenging problems in criminalistics. In many cases, it can be unbelievably valuable information, providing tools that will nourish the court's conviction regarding participation or elimination of potential suspects, reducing the time spent in the investigative process, minimizing errors during the prosecution and allowing the correct application of duties, rights and sanctions $[5,6,8]$. Therefore, the methods development that can estimate latent fingermark aging may represent an improvement in forensic procedures [9].

Specifically to fingermarks, which are physical evidence formed by biological materials, there are three main factors that influence the temporal analysis: (1) conditions inherent to the donor of the fingermark; (2) transfer conditions (deposition and the substrate or support); (3) environmental conditions in which the evidence is exposed. The time lapse determination between the deposition of the fingermark and its analysis will be the result of the complex and dynamic interaction of all these variables [7].

Different analytical methods have been employed to aging and chemical profile studies, such as gas chromatography, mass spectrometry and spectroscopy methods - Infrared and Raman [5-8]. Among these, spectroscopy methods are mostly non-destructive, low cost and quick analysis time (seconds) [10] and when it is combined with microscopy can provide good results in fingermark studies $[9,10]$. Spectroscopic methods, especially infrared, have been applied to characterize the composition of fingermarks, specifically the lipid components [11]. The Fourier Transform Infrared Microscopy ( $\mu$-FTIR) method is portrayed in the literature as the most common spectroscopic technique for fingermark analysis [12]. The operation of the $\mu$-FTIR in atmospheric conditions is a significant attribute for the study of latent fingermarks, since compositional or morphological changes can be observed on different surfaces both as a function of the time elapsed after deposition and according to the environmental conditions [11,12].

The practical implementation in the forensic routine of aging fingermark studies is still put to the test. For forensic practices, the time estimation of fingermarks can change the course of an investigation. Weynermann et al., (2011), developed as the main strategy for the improvement of fingermark aging methods, the selection of a chemical target present in the sample that changes over time, but being reproducible and measurable [5]. Focusing on the analysis of lipids, one of the principal classes of compounds of interest in aging studies, the characterization of di- and triglycerides demonstrated rapid degradation over time and are frequently present in this type of sample $[13,14,15]$.

Spectroscopic methods are intrinsic to the subsequent use of chemometrics, generating results that can increase the correlation between samples [11]. Multivariate data analysis (MDA) involves many tools, like variables selection using the ant of colony algorithm (AOC) genetic algorithm (GA) and one of the supervised methods of pattern recognition, like Linear Discriminant Analysis (LDA), that select the information on infrared spectra and describing relationships between samples and variables.

Therefore, this work developed a preliminary study for aged fingermarks analysis using $\mu-F T I R$ and chemometric tools for analysis optimization and pattern recognition between samples.

\section{MATERIALS AND METHODS}

\section{Latent Fingermarks Deposition and Development}

Latent fingermarks were collected from three Caucasian female donors, aged 30-34 years with a typical diet and without using cosmetics to minimizes the chance of anomalous results [16] Donors performed 
their daily activities, the only condition where they did not wash their hands 45 minutes before collection. In moments before the collection, donors were asked to rub their fingers on the forehead and nose $[7,16]$. The deposition protocol comprised the collection of the fingermark of the right thumb on a reflective microscope slide provided by Agilent Technologies, previously cleaned with ethanol, exerting a force between 1.0 and $1.5 \mathrm{~kg}$ for 15 seconds. Two samples were collected, one from the right thumb and other from the right index finger.

To simulate real materials that are collected at crime scenes, different deposition surfaces was tested like: soda can; glass slide covered with self-adhesive film; candy packaging; silver tape; stiletto blade; aluminum foil; Tetra Pak ${ }^{\circledR}$ box.

\section{Kinetic Conditions}

The monitored times in the analysis were: hour zero, 3 days (72 hours), 4 days (96 hours), 5 days (120 hours) and 6 days (144 hours). It is described in the literature that the most significant chemical changes in fingermarks occur in the first week after deposition. The choice of short intervals was made to observe these changes $[12,16]$. The samples were kept at room temperature with daily monitoring and exposed to light. All analyzes were performed in the morning, five spectra were collected per donor and three spectra were selected, considering the best quality of the collection, with less external interferences and noise. Zero-time analyzes were performed up to one hour after the first collection. The analysis region was observed in the microscope connected to the equipment, choosing five random points on the fingermark ridges as shown at Figure 1.

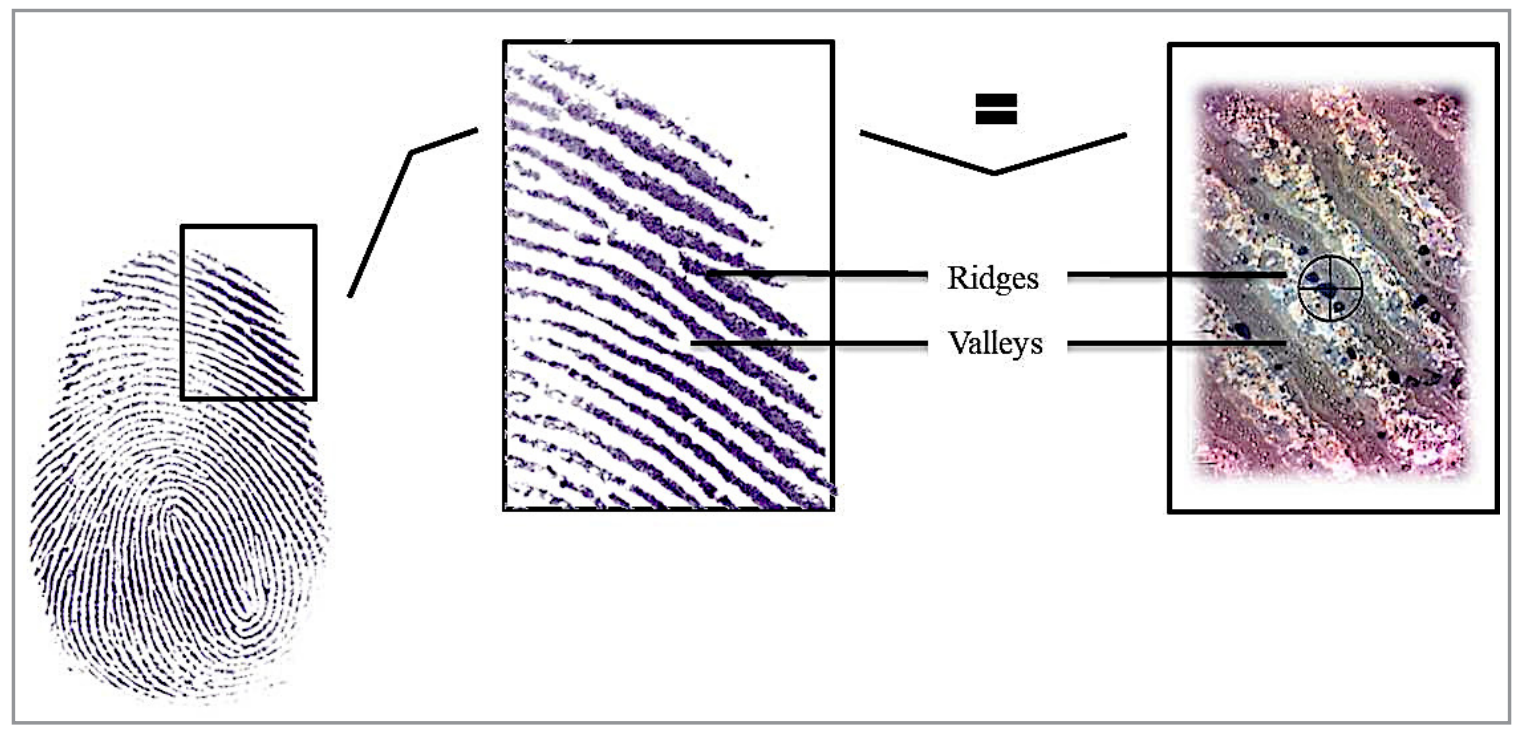

Figure 1. Visualization of fingerprint ridge details with naked eye and with microscopy $(1: 500 \mu \mathrm{m})$.

\section{Equipment and Method Conditions}

The analyzes were performed on the Cary 630 FTIR Spectrometer (Agilent Technologies - Santa Clara, CA - USA) with Survey IRTM Infrared Microspectroscopy Accessory (Czitek-Danbury, CT - USA). 320 scans were collected per sample at a resolution of $16 \mathrm{~cm}^{-1}$ with a spectral range of $4000-650 \mathrm{~cm}^{-1}$. The aperture of the accessory lens was $200 \mu \mathrm{m}$ and infrared mode in reflection and Y-Axis unit in absorbance. All samples were visualized, the analysis points were photographed, and the infrared spectra were collected using MicroLab FTIR Software (Agilent Technologies - Santa Clara, CA - USA). Background collection was carried out at each analysis to minimize external influences and level the conditions of each sample. 


\section{Chemometric Tools}

The Matlab Version 7.10.0.499 software (R2010a - MathWorks Inc. Natick, MT, USA) [17] was used with the Kennard-Stone algorithm [18]; the routines of: PCA Toolbox 1.5 (Milano Chemometrics and QSAR research Group) [19]; Ant of Colony (AOC); Genetic Algorithm (GA) and Linear Discriminant Analysis (LDA) Variable Selection Toolbox [20].

The Kennard-Stone algorithm selects samples with a uniform distribution, starting with a selection of two samples with large Euclidean distance in a sample space. For each of the remaining samples, the lower distance is calculated with respect to the samples already selected. Then, the sample with the smaller distance is retained, and the procedure is repeated until a certain number of samples is selected [18].

It was used a supervised method to perform the samples classification. Due to the large number of spectral variables, it was necessary to apply a variable selection tool. Among the available resources in the software, the GA was chosen for being a classic tool while the AOC is an emerging tool, both consolidated in chemometrics studies [21].

The GA is an adaptation of the evolutionary systems of natural selection developed by Darwin applied as a resource in mathematical and computational tools. The principle of GA is to create a primordial set with several possible solutions to the problem. This set is called the initial population and each solution belonging to this population is called an individual. In this case, the genetic material is a chromosome, and this is an individual itself. This codification of individuals aims to enable the application of genetic operators and the concept of natural selection on existing solutions, thus being able to combine their genetic materials, searching for more and more adapted individuals over several generations, that is, solutions with a lower degree of error for the problem addressed [21]. The AOC is an algorithm inspired by the cooperative behavior of real ant of colonies, which search for the best path (shorter distances and fewer obstacles) between the colony and a food source, coordinated by pheromones. This tool has been successfully employed as a powerful resource for selecting variables from chemical data in multivariate calibration, with linear discriminant analysis (LDA) [21].

The LDA is a linear combination of original characteristics of the sample set which is characterized by producing the maximum separation between two populations. And its main objectives are to verify if the groups are correctly discriminated, to classify unknown observations and to verify which are the most important variables for the discrimination of these groups [19,21]. This tool takes a different approach in that it considers the existence of classes for the data; projecting the probability distribution of the data on the axes, and therefore not only maintains but highlights a linear separation of the data if it exists [19,21].

\section{RESULTS AND DISCUSSION Spectroscopy Findings}

First, it was select the spectra region of analysis choosing the region between 1749 and $649 \mathrm{~cm}^{-1}$ totalizing 150 variables. This region was selected because it contains characteristic information about the sample, called fingerprint region, and other relevant signals for this study. The region selection is also necessary for limiting variables during the process of understanding the results, focusing on the recognition of patterns in the sample [22] The spectra were normalized between 0 and 1 (Figure 2). The normalization pre-processing step was used to transform the original data into an appropriate model for dataset processing, performed by scaling values in the indicated range [23,24].

It should be noted some limitations of the analytical tool, only fingermarks deposited on the company's microscopy slide showed spectra with adequate resolution for identifying bands. There was also a limitation regarding the image resolution, not being possible to observe the entire fingermark in a widefield microscopy. Therefore, it was not possible to view a complete fingermark image or make an automatic point selection for spectral analysis. 


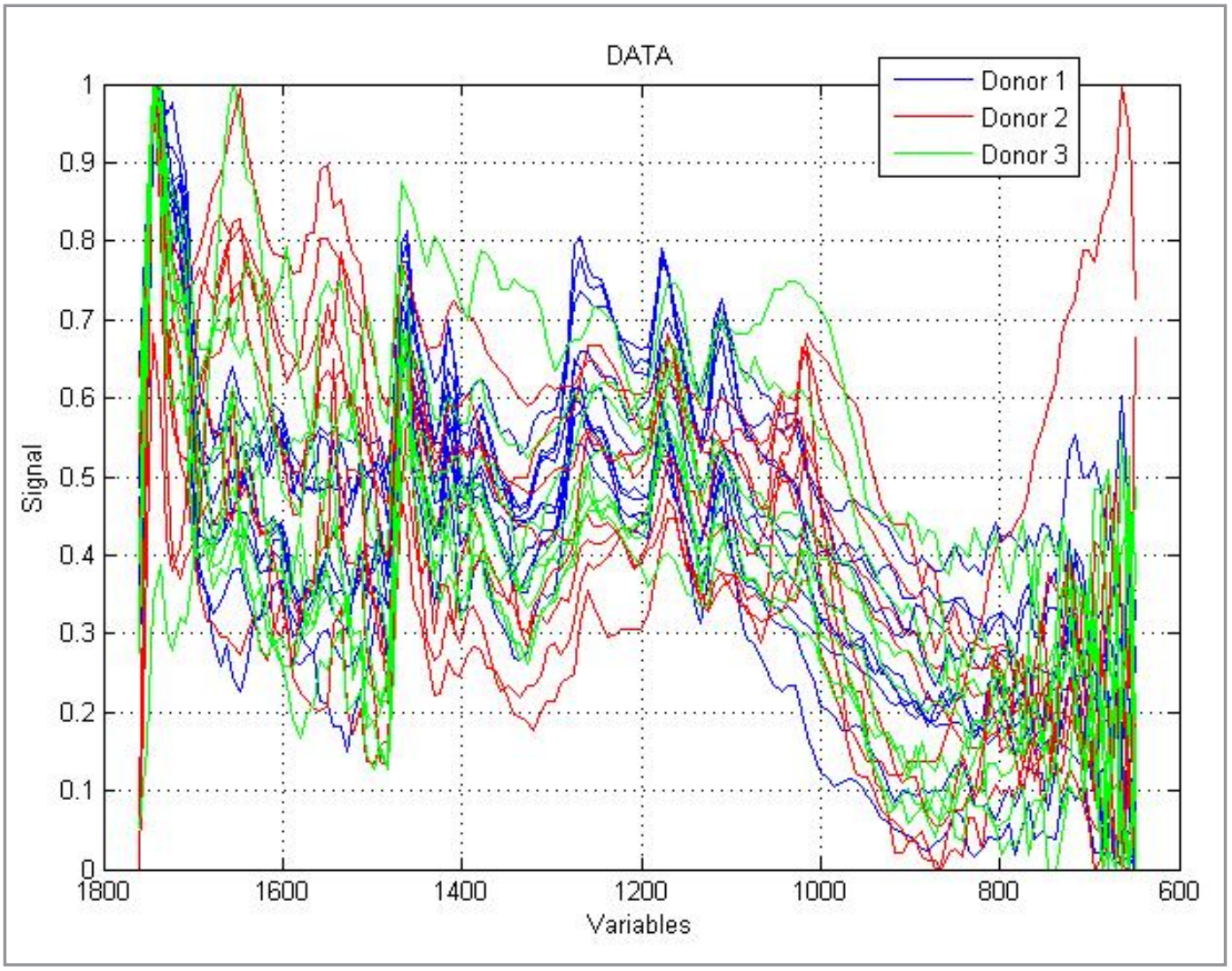

Figure 2. Normalized spectra with the selected fingerprint region.

Studies show that the infrared bands of fingermarks are similar between donors [5-7,25-28] as can be seen in Figure 3, corresponding to the overlay spectra of the three donors and their microscopic images.

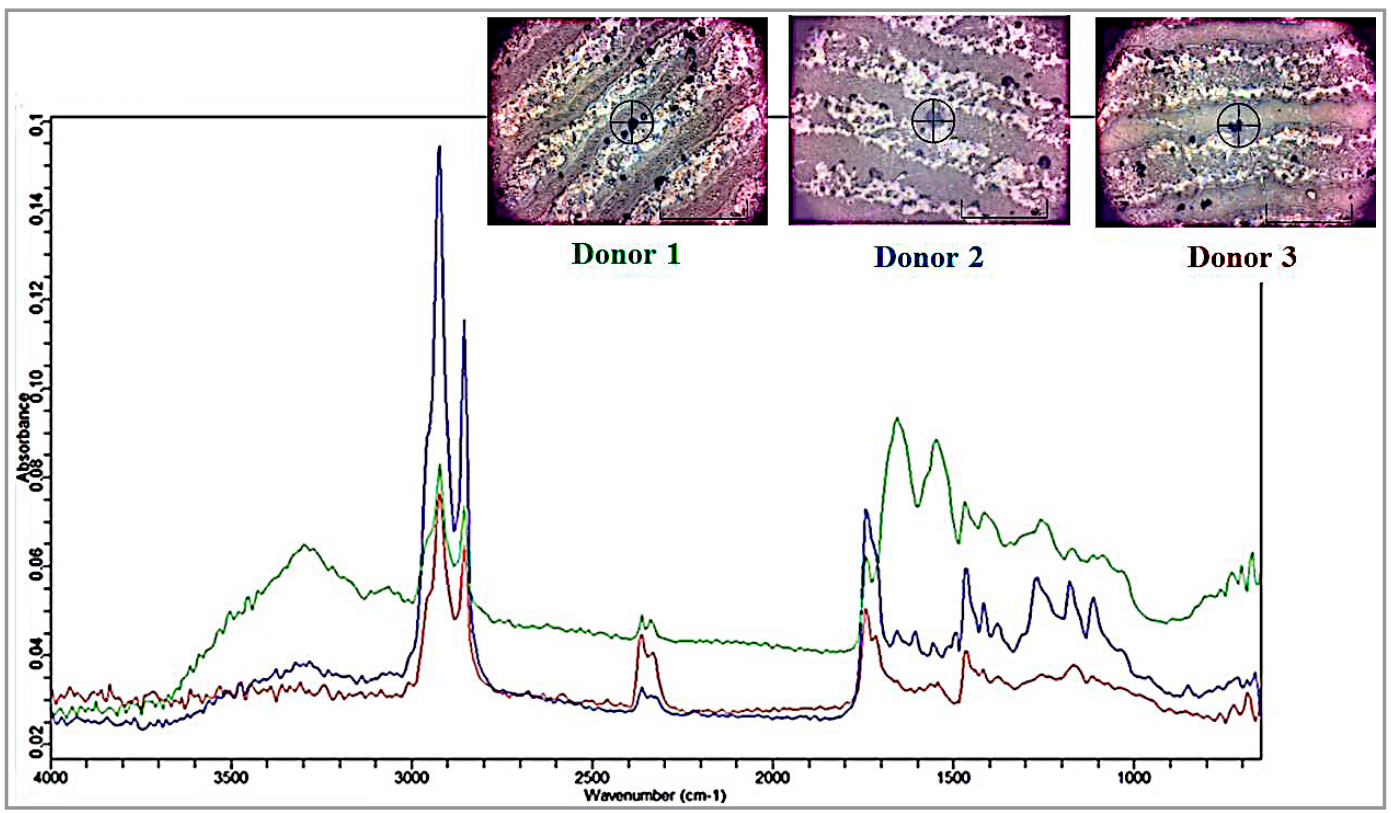

Figure 3. Comparison of spectra of the three donors with visualization of the microscopic region with an increase of $200 \mu \mathrm{m}$ of selected analysis. 
The signals correspond mainly to the major functional groups that form wax esters and fatty acids. Figure 4 summarizes the main signs observed in the standard spectrum. In the samples collected from the three donors, it was not possible to evidence eccrine secretions, as there are no signs that indicate vibrations of secondary amides, coming from proteins. According to Girod et al. (2015), eccrine secretions are represented by $\mathrm{N}-\mathrm{H}$ stretches in the regions of 3200 and $1500 \mathrm{~cm}^{-1}$ [16], which is not reported in our study. In fact, it is more common to find patterns of spectra corresponding only to sebaceous secretions $[12,16]$, as evidenced in our samples. In all samples collected, it was possible to see a pattern in the spectrum in which there are two more informative absorbance regions, with characteristic vibrations indicative of the presence of lipids because they have signs of saturated esters. The first region being $1800-1100 \mathrm{~cm}^{-1}$ and the second region being $3000-2800 \mathrm{~cm}^{-1}$.

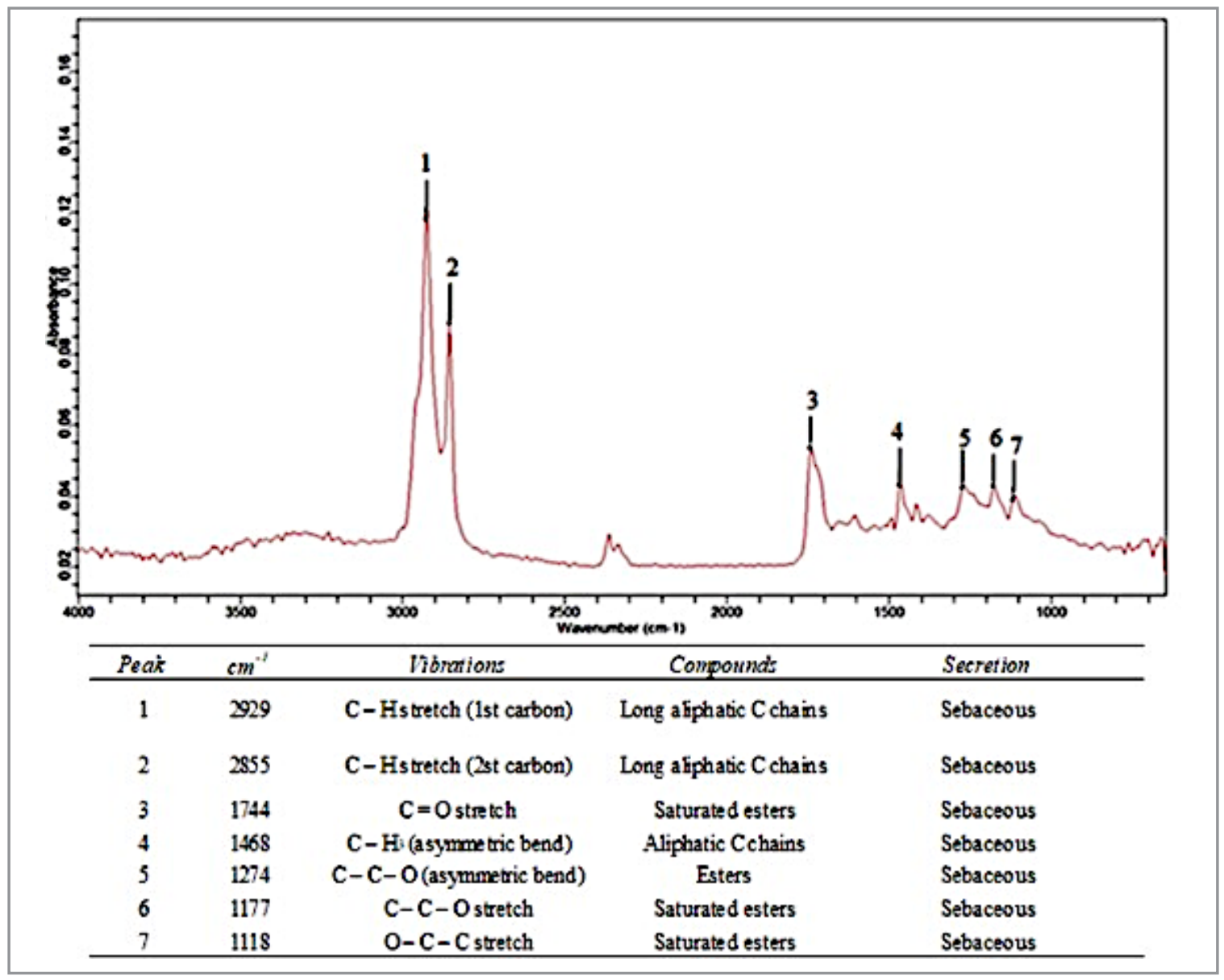

Figure 4. Fresh fingermark sample spectrum containing sebaceous material with main vibrational bands identified in the table $[12,16]$.

The tests carried out on the materials to simulate different surfaces was inconclusive. All materials allowed the equipment to focus on the sample, making a clear image and allowing the colection of the background stage prior to each analysis. However, the materials had different roughness, making it difficult to locate the region of interest for analysis; thus, the infrared spectrum was full of noise, with baseline elevation and overlapping signals. Therefore, only the reflective slide provided by the equipment company resulted in a possible image to identify the fingermarks and the region of interest for analysis. Despite differences in the amount of material deposited by the 3 donors, it is possible to see characteristic signs pattern among the donor's samples. 


\section{Chemometric Findings}

The Kennard-Stone algorithm was applied to divide the samples into the training and test sets in order to assess whether variable selection models would be applicable to the samples. Then, each variable selection model was tested five times in which the test set was tested against the model. Figures 5 show the regions of the spectrum selected by AOC and GA algorithms for donor and sample separation, respectively. It was acchieve better separation of the sample set in relation to the test set. Some regions chosen in the two models are similar, however, it was observed that the region with less intense signals is not chosen by the AOC model, which may suggest a performance slightly lower than the GA. The results were organized below according to the sample separation between time and donors.
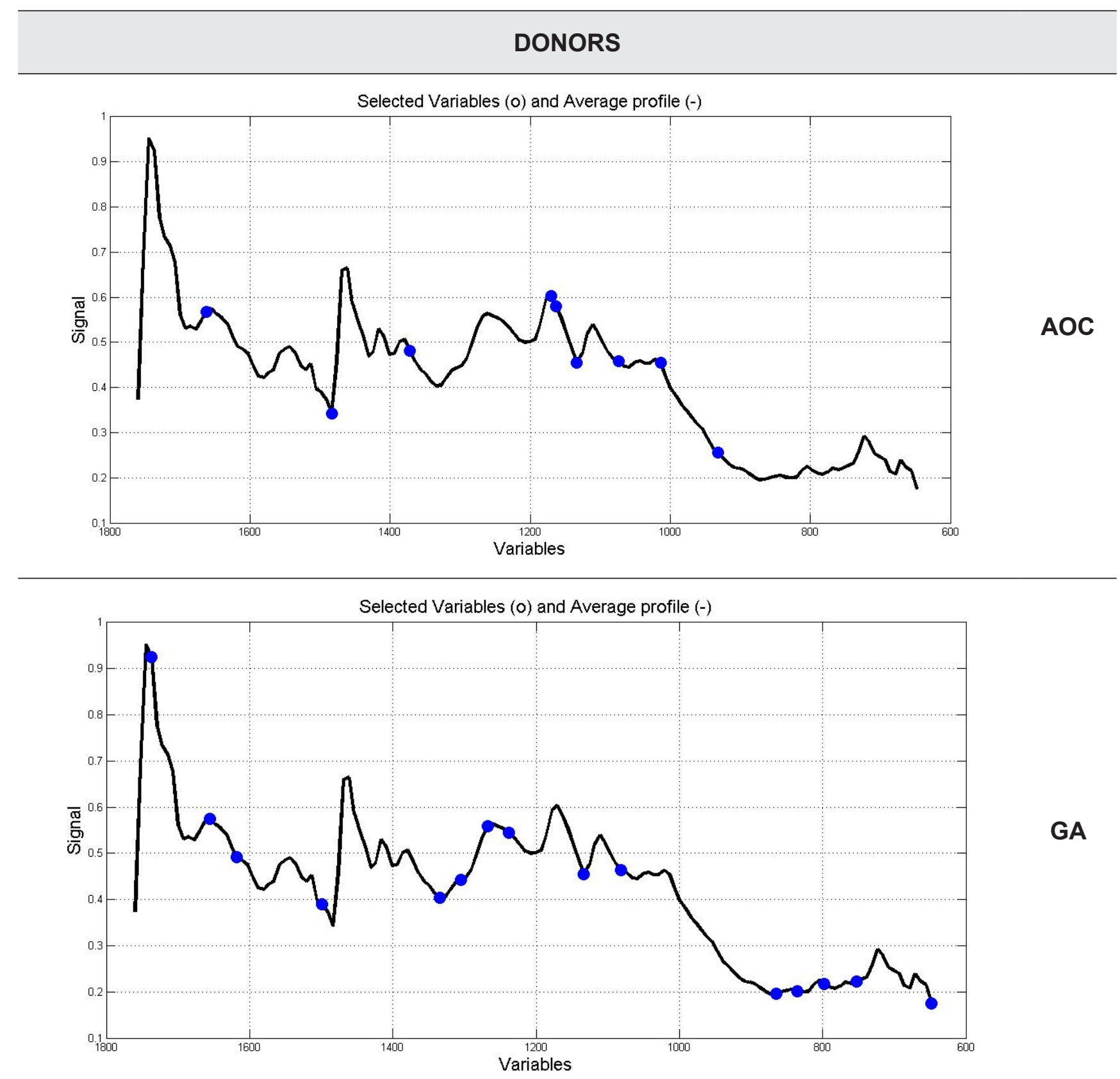

GA

Figure 5. Regions of interest responsible for sample separation scores. 


\section{TIME}
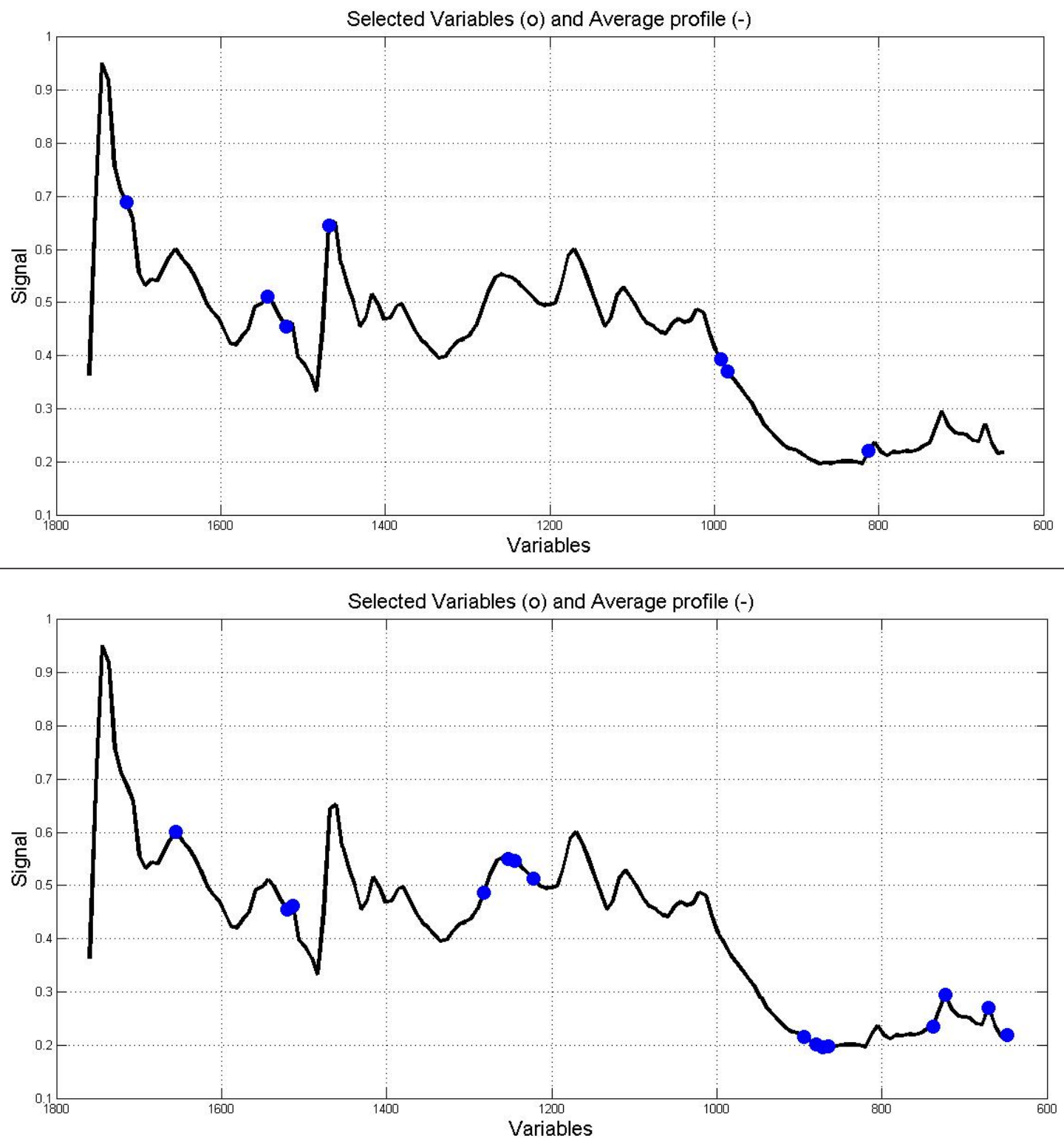

GA

Figure 5. Regions of interest responsible for sample separation scores. (Continuation)

\section{Donor Separarion Results}

Comparing the results of AOC and GA with subsequent application of the LDA (Figure 6) it is possible to observe a clear sampling separation of both the training set and the test set for the three different donors. Thus, with these tools, it was possible to see that even with a small amount of samples the differentiation of classes (donors and time) could be performed. With regard specifically to fingermark samples, it is considered impossible to conduct an aging model that works for all types of fingerprints because of the great variability between different donors and the same donor $[5,6,11,16]$. Thus, Girod et al. (2015) suggested the construction of a new model in order to study from the initial composition to the aging of the sample from a single donor [16]. Based on this idea, we use only three female donors, because female samples tend to have greater intra and inter donor variation (make up and cosmetics uses and hormonal 
differences). It can be seen in Figure 6 that the prediction samples (stars) are slightly apart compared to the training samples (balls). In both results only a sample of the test group is shifted in the graphs, this shows that in terms of responses the methods are equivalent. In addition, GA is a slightly better model, but there is no greater representativeness than the point of discarding the AOC model.
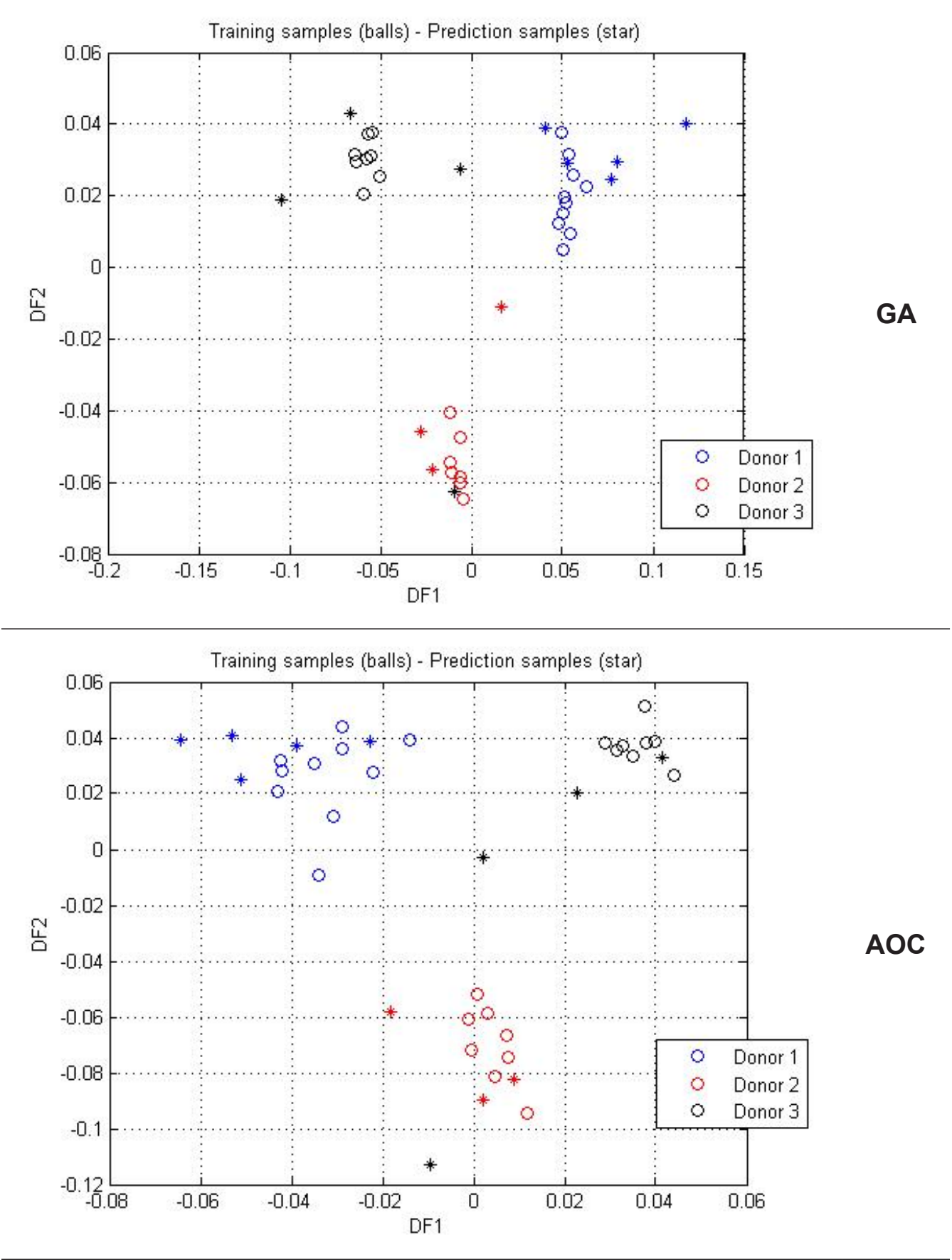

Figure 6. Results of sample separation by GA and AOC models.

\section{Time Separation Results}

One of the forms to see fingermarks aging is to follow the time evolution of the sample. According to the number of samples available, only four of the six times analyzed were used to create the test set and 
training set. The result was similar for both GA and AOC, in which, even with a small number of samples, it was possible to observe a trend of separation, as shown in Figure 7 . Thus the application of this model can provide consistent results if applied to a larger number of samples.

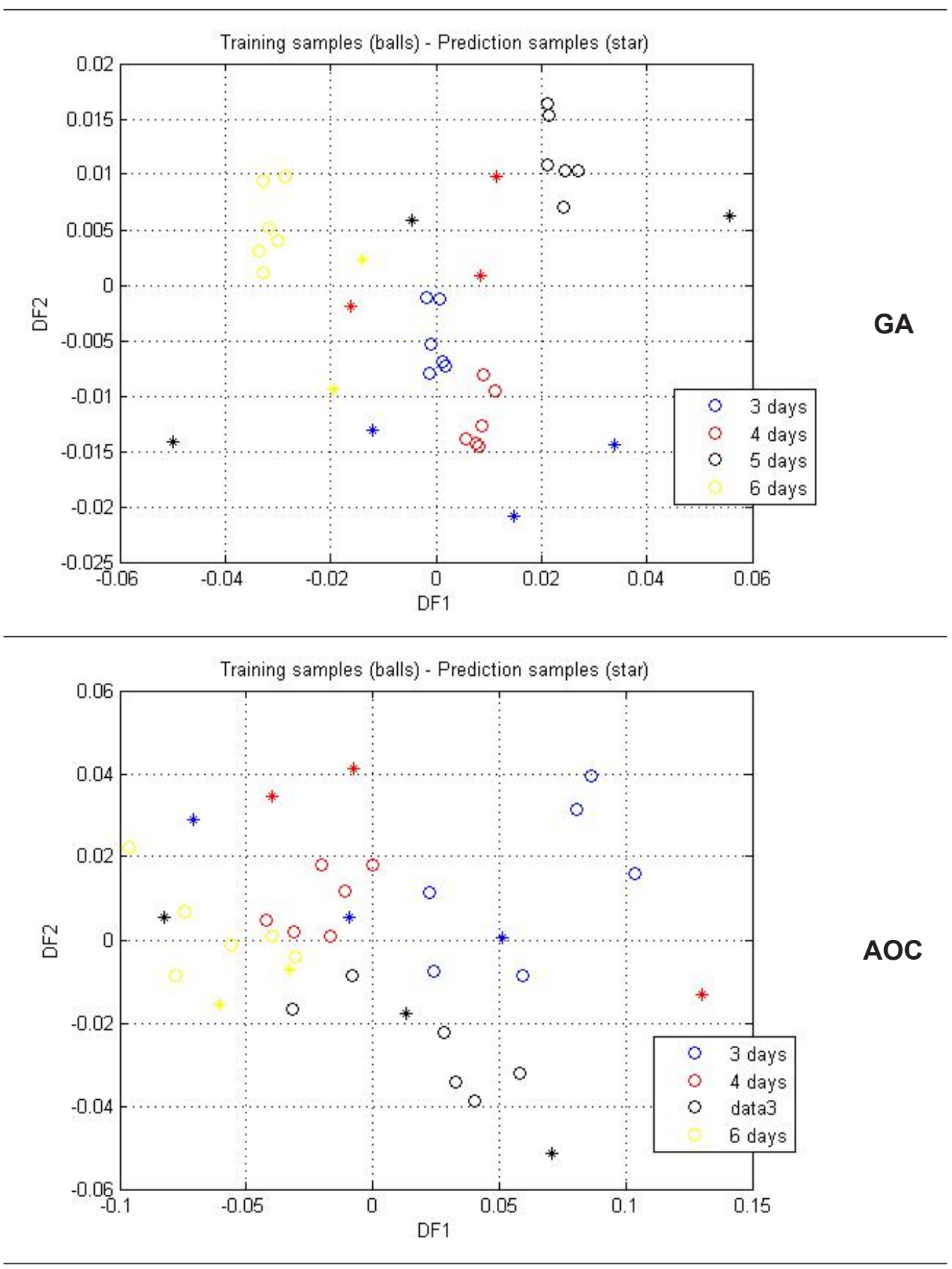

Figure 7. Samples separation in four days.

The spectra of aged fingermarks originally containing mainly lipid compounds (i.e., sebaceous secretions) showed a decrease in the intensity of all vibrational bands. According to Girod et al. (2015) study, fingermarks could be grouped by age, but the aging profile was significantly influenced by storage conditions and also by substrate when specimens were stored in the dark [16]. 
Table I shows the performance of the models for the separation between donors and the analysis times. The classes are the sample categories, being three donors and four times evaluated, excluding the test set. Precision expresses the agreement degree between the results of a series of measurements carried out for the same homogeneous sample under determined conditions [29-31] and it was calculated according the Equation 1, for each class. The sensitivity, samples belonging to the class and classified correctly in this class, were considered for the evaluation of the classification achieved with the multivariate methods and was calculated to Equation 2. The specificity, samples not belonging to the modeled class and correctly classified as not belonging, was calculated according to Equation 3:

$$
\begin{aligned}
\text { Precision } & =\frac{T P}{T P+F P} \times 100 \\
\text { Sensitivity } & =\frac{T P}{T P+F N} \times 100 \\
\text { Specificity } & =\frac{T N}{T N+F P} \times 100
\end{aligned}
$$

where TP is the number of true positives; TN is true negatives; FP is false positives and FN is false negatives [29-31]. It is possible to observe the performance of the models proved to be superior for sample separation by donor, due to greater accuracy and less errors. However, although there is no clear division

\begin{tabular}{|c|c|c|c|c|c|c|c|c|c|c|c|c|c|c|}
\hline \multirow{2}{*}{$\begin{array}{l}\text { Samples } \\
\text { Variable Selection }\end{array}$} & \multicolumn{6}{|c|}{ Donors } & \multicolumn{8}{|c|}{ Time } \\
\hline & & GA & & & AOC & & \multicolumn{4}{|c|}{ GA } & \multicolumn{4}{|c|}{$\mathrm{AOC}$} \\
\hline Accuracy rate (\%) & & 93.98 & & & 91.67 & & \multicolumn{4}{|c|}{65.97} & \multicolumn{4}{|c|}{77.43} \\
\hline Misclassification rate (\%) & & 6.01 & & & 6.02 & & \multicolumn{4}{|c|}{34.02} & \multicolumn{4}{|c|}{22.56} \\
\hline Total errors & & 1 & & & 1 & & \multicolumn{4}{|c|}{6} & \multicolumn{4}{|c|}{4} \\
\hline Class & 1 & 2 & 3 & 1 & 2 & 3 & 1 & 2 & 3 & 4 & 1 & 2 & 3 & 4 \\
\hline Precision & 1 & 0.75 & 1 & 1 & 0.75 & 1 & 0.5 & 0.5 & 0.33 & 0.5 & 0.5 & 0.66 & 1 & 0.5 \\
\hline Sensitivity & 1 & 1 & 0.75 & 1 & 1 & 0.5 & 0.33 & 0.33 & 0.33 & 1 & 0.33 & 0.66 & 0.66 & 1 \\
\hline Specificity & 1 & 0.88 & 1 & 1 & 0.88 & 1 & 0.87 & 0.87 & 0.75 & 0.77 & 0.87 & 0.87 & 1 & 0.77 \\
\hline
\end{tabular}
as to the analysis times, it is possible to see a homogeneity of the classes in the samples for time analysis.

Table I. Performance of selected models for separating samples into donors and time 
The fingermarks samples have great variability between donors [4-7], so, it was decided to carry out the pilot study with a small number of donors in order to establish a method that could be reproduced in the forensic routine. Thus, an preliminary aging model was built with 3 donors of the same sex to verify whether samples would be separated without directly considering gender as a variant. The use of the $\mu$-FTIR method provided comparable spectra in a short period of analysis. The chemometric tools allowed to see that in a single infrared spectrum collected in the described parameters it contains thousands of variables that, although correlated, much information does not necessarily explain a significant variance related to differentiation by time, environmental exposure or effects of the sample itself. Therefore, the selection of variables performed by chemometrics was important [30].

The use of AOC and GA reduced the data set, selecting the relevant variables and the LDA allowed the separation of samples by time. Even for a period of six days, it was possible to observe that the most important transformations occurred in the samples from time zero to $72 \mathrm{~h}, 120 \mathrm{~h}$ and $144 \mathrm{~h}$, as observed in Figure 8. This opens the possibility of sparing the analyzes for the next studies.

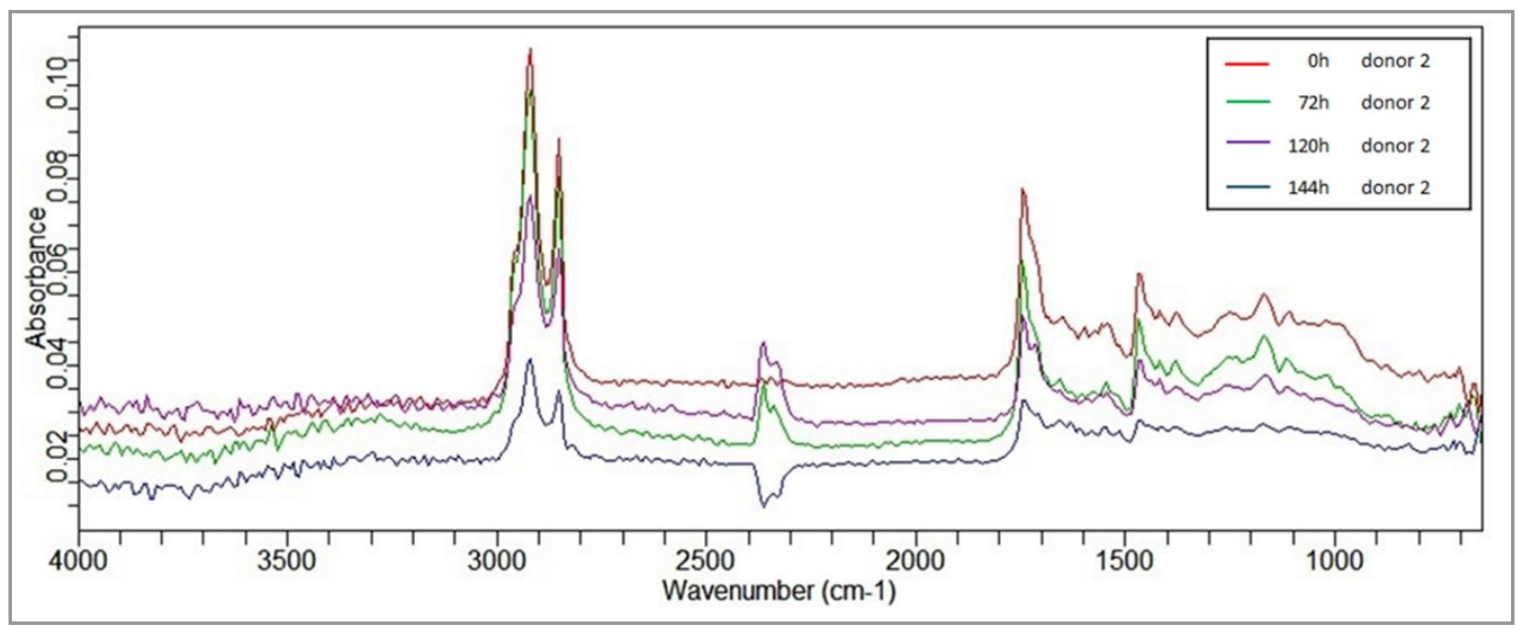

Figure 8. Overlaid samples of donor 2 in aging parameters. The inversion of bands shown in the middle of the spectrum, between $2300-200 \mathrm{~cm}^{-1}$, corresponds to $\mathrm{CO}_{2}$. These changes can occur in the spectrum because the sample is not subjected to vacuum during an analysis, thus, the spectrum can undergo changes according to the instability of the environment.

The limiting steps of the study are: (i) it was not possible adapted the method for other surfaces of sample collection; (ii) need to increase the number of the samples and expand to both genders to be more representative; (iii) need to adapted to be used in real cases.

In general, it was possible to observe a pattern in the fingerprint spectra analyzed by $\mu$-FTIR corresponding to sebaceous components of the samples. For a period of six days it was possible to see an decreasing intensity of the compounds as the days passed, suggesting a characteristic degradation capable of being observed in studies related to fingermark aging analysis.

\section{CONCLUSIONS}

A preliminary fingermark study was carried out with three donors in order to establish a sample aging profile to suggest a temporal estimate of the forensic evidence. The $\mu$-FTIR method enabled a fast and non-destructive analysis, but with some but with some limitations concerning about the surface were fingermark can be analysed. It was possible to verify the presence of sebaceous components that had degradation by decreasing the signal in the infrared spectrum in the period of six days and also evidenced by the use of the GA and LDA chemometric tools. In addition, it was possible to separate donors, even with a small number of samples and a short study interval. The $\mu-F T I R$ method linked to the use of 
chemometrics is promising for the reality of the forensic expertise in Brazil and is already consolidated in other countries, but it needs more concrete studies to be placed in the forensic routine as an additional resource in the forensic identification protocols.

\section{Conflicts of interest}

The authors declare that there is no conflict of interest. This work was supported by "Fundo de Amparo à Pesquisa do Rio Grande do Sul" (FAPERGS) - fund number 17/2551-0000839-1 and by National Institute of Science and Technology (INCT Forensics - CNPq 465450/2014-8).

\section{Acknowledgements}

This work was supported by: "Fundo de Amparo à Pesquisa do Rio Grande do Sul" (FAPERGS) - grant $n^{\circ}$ 17/2551-0000839-1; National Institute of Science and Technology (INCT Forensics - CNPq 465450/20148; "Conselho Nacional de Desenvolvimento Científico e Tecnológico" (CNPq) and "Coordenação de Aperfeiçoamento de Pessoal de Nível Superior" (CAPES) - fund n 88882.345923/2019-01.

\section{REFERENCES}

1. Mariotti, K. C. Brazilian Journal of Forensic Sciences, Medical Law and Bioethics, 2020, 9 (2), pp 210-228 (http://dx.doi.org/10.17063/bjfs9(2)y2020210).

2. Peterson, J.; Sommers, I.; Baskin, D.; Johnson, D. The Role and Impact of Forensic Evidence in the Criminal Justice Process. US Department of Justice, Washington DC, 2010, Chapter 2, p 14.

3. Girod, A; Spyratou, A; Holmes, D.; Weyermann, C. Sci Justice, 2016, 56 (3), pp 165-180 (http://dx.doi. org/10.1016/j.scijus.2015.12.004).

4. Girod, A.; Ramotowski, R.; Lambrechts, S.; Misrielal, P.; Aalders, M.; Weyermann, C. Forensic Sci. Int., 2016, 262, pp 212-226 (http://dx.doi.org/10.1016/j.forsciint.2016.03.021)

5. Weyermann, C.; Roux, C.; Champod, C. J. Forensic. Sci., 2011, 56 (1), pp 102-108 (http://dx.doi. org/10.1111/j.1556-4029.2010.01523.x).

6. Weyermann, C.; Ribaux, O. Sci. Justice, 2012, 52 (2), pp 68-75 (http://dx.doi.org/10.1016/j. scijus.2011.09.003).

7. Girod, A.; Ramotowski, R.; Weyermann, C. Forensic Sci. Int., 2012, 223 (1-3), pp 10-24 (https://doi. org/10.1016/j.forsciint.2012.05.018).

8. De Alcaraz-Fossoul, J.; Patris, C. M.; Freixat, C. B.; McGarr, L.; Brandelli, D.; Stow, K.; Badia, M. G. J. Forensic. Sci., 2016, 61 (2), pp 322-333 (http://dx.doi.org/10.1111/1556-4029.13007).

9. Barros, R. M.; Faria, B. E. F.; Kuckelhaus, S. A. S. Sci. Justice, 2013, 53 (4), pp 402-408 (http://dx.doi. org/10.1016/j.scijus.2013.08.002).

10. De Alcaraz-Fossoul, J.; Feixat, C. B.; Tasker, J.; McGarr, L.; Stow, K.; Carreras-Marin, C.; Oset, J. T.; Badia, M. G. J. Forensic. Sci., 2016, 61 (4), pp 947-958 (http://dx.doi.org/10.1111/1556-4029.13099).

11. Girod, A.; Ramotowski, R.; Lambrechts, S.; Misrielal, P.; Aalders, M.; Weyermann, C. Forensic Sci Int., 2016, 262, p 212.

12. Johnston, A.; Rogers, K. Sci. Justice, 2018, 58 (2), pp 121-127 (https://doi.org/10.1016/j. scijus.2017.11.004).

13. Cadd, S.; Islam, M.; Manson, P.; Bleay, S. Sci. Justice, 2015, 55 (4), pp 219-238 (http://dx.doi. org/10.1016/j.scijus.2015.02.004).

14. Frick, A. A.; Weyermann, C. Analyst, 2019, 144 (11), pp 3590-3600 (http://dx.doi.org/10.1039/ c9an00521h).

15. Pleik, S.; Spengler, B.; Bhandari, D. R.; Luhn, S.; Schäfer, T.; Urbach, D.; Kirsch, D. Analyst, 2018, 143 (5), pp 1197-1209 (http://dx.doi.org/10.1039/c7an01506b).

16. Girod, A.; Xiao, L.; Reedy, B.; Roux, C. Forensic Sci. Int., 2015, 254, pp 185-196 (http://doi.org/10.1016/j. forsciint.2015.07.022).

17. https://www.mathworks.com/products/matlab.html [Accessed 21 June 2020]. 
18. Kennard, R. W.; Stone, L. A. Technometrics, 1969, 11 (1), pp 137-148 (https://doi.org/10.1080/00401 706.1969.10490666).

19. Ballabio, D. Chemom. Intell. Lab. Syst., 2015, 149 (Part B), pp 1-9 (https://doi.org/10.1016/j. chemolab.2015.10.003).

20. Homemade routine available under request to araujo.gomes@ufrgs.br

21. Consonni, V.; Baccolo, G.; Gosetti, F.; Todeschini, R.; Ballabio, D. Chemom. Intell. Lab. Syst., 2021, 213 (https://doi.org/10.1016/j.chemolab.2021.104313).

22. Smith, B. C. Fundamentals of Fourier transform infrared spectroscopy, $2^{\text {nd }}$ ed., CRC Press, 2011, Chapter 1, p 10.

23. Ferreira, M. M. C. Quimiometria: Conceitos, Métodos e Aplicações. Editora UNICAMP, Campinas, 2016, Capítulo 5, p 409.

24. Pontes, A. S.; Araújo, A.; Marinho, W.; Diniz, P. H. G. D.; Gomes, A. A.; Goicoechea, H. C.; Silva, E. C.; Araújo, M. C. U. J. Chemom., 2020, 34 (12) (https://doi.org/10.1002/cem.3292).

25. Kumar, R.; Sharma, V. TrAC, Trends Anal. Chem., 2018, 105, pp 191-201 (https://dx.doi.org/10.1016/j. trac.2018.05.010).

26. Champod, C. Fingerprints and Other Ridge skin Impressions. $2^{\text {nd }}$ Ed. CRC Press, Boca Raton, 2016, Chapter 4, p 119.

27. Frick, A. A.; Fritz, P.; Lewis, S. W. Chemistry of Print Residue. In: Siegel, J. A.; Saukko, P. J.; Houck, M. M. (Eds). Encyclopedia of Forensic Sciences (2 ${ }^{\text {nd }}$ Edition). Academic Press, Waltham, 2013, pp 92-97 (https://doi.org/10.1016/B978-0-12-382165-2.00262-2).

28. Francese, S.; Bradshaw, R.; Ferguson, L. S.; Wolstenholme, R.; Clench, M. R.; Bleay, S. Analyst, 2013, 138, pp 4215-4228 (https://doi.org/10.1039/C3AN36896C).

29. Grasel, F. S.; Marcelo, M. C. A.; Ferrão, M. F. RSC Adv., 2016, 6, pp 32358-32364 (http://dx.doi. org/10.1039/c6ra00900j).

30. Grasel, F. S.; Ferrão, M. F. Anal. Methods, 2016, 8, 644 (http://dx.doi.org/10.1039/c5ay02526e)

31. Valderrama, P.; Braga, J. W. B.; Poppi, R. J. Quim. Nova, 2009, 32 (5), pp 1278-1287 (http://dx.doi. org/10.1590/S0100-40422009000500034). 